\title{
Non-Markovian dynamics of fully coupled fermionic and bosonic oscillators
}

\author{
V. V. Sargsyan, ${ }^{1}$ D. Lacroix, ${ }^{2}$ G. G. Adamian, ${ }^{1}$ and N. V. Antonenko ${ }^{1,3}$ \\ ${ }^{1}$ Joint Institute for Nuclear Research, 141980 Dubna, Russia \\ ${ }^{2}$ Institut de Physique Nucléaire, IN2P3-CNRS, Université Paris-Sud, F-91406 Orsay Cedex, France \\ ${ }^{3}$ Mathematical Physics Department, Tomsk Polytechnic University, 634050 Tomsk, Russia
}

(Received 21 July 2016; published 17 March 2017)

\begin{abstract}
The non-Markovian Langevin approach is applied to study the dynamics of fermionic (bosonic) oscillator linearly coupled to a fermionic (bosonic) environment. The analytical expressions for occupation numbers in two different types of couplings (rotating-wave approximation and fully coupled) are compared and discussed. The weak-coupling and high- and low-temperature limits are considered as well. The conditions under which the environment imposes its thermal equilibrium on the collective subsystem are discussed. The sameness of the results, obtained with both the Langevin approach and the discretized environment method are shown. Shortand long-time nonequilibrium dynamics of fermionic and bosonic open quantum systems are analyzed both analytically and numerically.
\end{abstract}

DOI: 10.1103/PhysRevA.95.032119

\section{INTRODUCTION}

A goal of nonequilibrium statistical mechanics is to make good, yet simple, models of complicated phenomena out of equilibrium and to analyze them. Investigations of dissipative quantum non-Markovian subsystems beyond the weakcoupling or high-temperature limits request exactly solvable models [1-24] to be widely applicable. This class of models has been considered to investigate various aspects in mesoscopic physics where a given two-level system interacts with a bosonic or fermionic environment modeled by a set of harmonic oscillators. It is convenient to describe an influence of the environment on the system using a spectral density which contains information about the spectrum of the environment as well as the frequency-dependent coupling $[11,12,19,25]$. The complete information about the effect of the thermal bath is encapsulated in the single spectral function $[1,2,7]$. The systems with fermionic baths are of interest up due to the possibility of creating and manipulating rather small fermionic systems in various fields of physics [7,11,12,17-19,26]. Models describing the interaction between fermionic systems and spin degrees of freedom play an important role [7,11,12,17-19,26]. In particular, an approach of these systems to equilibrium would greatly help to understand how they can reach the thermodynamic limits and how the thermalizations of the isolated system and the collective subsystem of this isolated system are related $[27,28]$. It is interesting to study the crossover from coherent to incoherent dynamics in the damped quantum system [29].

Recently, several stochastic methods have been proposed to consider the problem of a system coupled to an environment. This includes the functional-integral approach $[7,11,12,17-$ $19,26]$, the quantum state diffusion approach [30-33], quantum jumps [34], the quantum Langevin approach [20-24,35], the quantum Monte Carlo approach [36-39], or the stochastic method of Refs. [19,40]. Applications of stochastic methods to non-Markovian open quantum systems are progressing but still remain tedious when the complexity of the system increases. So, further development of theoretical methods is required.

In Ref. [41], we considered the quadratic fermionic Hamiltonians for collective and internal subsystems linearly coupled within the rotating-wave approximation (RWA) to analyze the role of the fermionic statistics (in the comparison with the bosonic statistics) in the dynamics of the collective motion. The Langevin approach [20-24] was applied to find the effects of fluctuations and dissipations in macroscopic systems. The Langevin method in the kinetic theory significantly simplifies the calculation of nonequilibrium quantum and thermal fluctuations and provides a clear picture of the dynamics. Many problems in various open quantum systems can be described by using the Langevin equations in the space of relevant collective coordinates. The results of the Langevin approach were confirmed by considering the dynamics of an open quantum system with the discretized environment method (DEM) [28] which allows us to incorporate environment explicitly in a discretized form.

The aim of the present work is to extent the results of Ref. [41] and consider the general case of a fully coupled (FC) oscillator modeling fermionic (bosonic) collective subsystem coupled with a fermionic (bosonic) heat bath. The results obtained will be checked with the DEM [28] extended to treat FC oscillator and compared with those obtained in the RWA case.

In Secs. II and III, the model is formulated and the expressions for occupation numbers are obtained. The asymptotic occupation numbers are discussed in Sec. IV. The weakcoupling limit is considered in Sec. V and the results are summarized in Sec. VI.

\section{MODEL HAMILTONIAN OF FULLY COUPLED FERMIONIC OSCILLATOR}

We consider a two-level fermionic system (collective subsystem) with creation $a^{\dagger}$ and annihilation $a$ operators, and with frequency $\hbar \omega$. This system interacts with a bath consisting of two-level fermionic systems, labeled by index $v$, with creation and annihilation operators $a_{v}^{\dagger}$ and $a_{v}$, and frequency $\hbar \omega_{v}$, respectively. For two-level fermionic systems, the operators satisfy the following permutation relations:

$$
\begin{aligned}
a a^{\dagger}+a^{\dagger} a & =1, \quad a a=a^{\dagger} a^{\dagger}=0, \\
a_{\nu} a_{v^{\prime}}+a_{v^{\prime}} a_{v} & =a_{v}^{\dagger} a_{v^{\prime}}^{\dagger}+a_{v^{\prime}}^{\dagger} a_{v}^{\dagger}=0, \\
a_{\nu} a_{v^{\prime}}^{\dagger}+a_{v^{\prime}}^{\dagger} a_{v} & =\delta_{v, v^{\prime}} .
\end{aligned}
$$


The Hamiltonian of the whole system is

$$
H=H_{s}+H_{b}+H_{c b}
$$

where

$$
H_{c}=\hbar \omega a^{+} a
$$

is the Hamiltonian of isolated collective subsystem, and

$$
H_{b}=\sum_{v} \hbar \omega_{\nu} a_{\nu}^{\dagger} a_{v}
$$

is the Hamiltonian of the bath. As the detailed analysis of the dynamics of occupation numbers in the case of RWA coupling between the system and bath was presented in Ref. [41], here we study the FC-type coupling. In this case, the interaction Hamiltonian is written as

$$
H_{c b}=\sum_{v} g_{v}\left(a^{\dagger}+a\right)\left(a_{v}^{\dagger}+a_{v}\right)
$$

The real constants $g_{v}$ in Eq. (5) determine the coupling strength between the collective and bath " $v$ " subsystems.

\section{EXPRESSIONS FOR OCCUPATION NUMBER OF COLLECTIVE SUBSYSTEM}

By commuting the creation and annihilation operators of the collective subsystem with total Hamiltonian $H$, one can obtain the Heisenberg equations of motion for corresponding operators:

$$
\begin{aligned}
\frac{d}{d t} a^{\dagger} & =i \omega a^{\dagger}+\left(1-2 a^{\dagger} a\right) \frac{i}{\hbar} \sum_{v} g_{v}\left(a_{v}^{\dagger}+a_{v}\right), \\
\frac{d}{d t} a & =-i \omega a-\left(1-2 a^{\dagger} a\right) \frac{i}{\hbar} \sum_{v} g_{v}\left(a_{v}^{\dagger}+a_{v}\right) .
\end{aligned}
$$

These equations contain terms proportional to $2 a^{\dagger} a$ and could not be solved analytically. However, keeping the terms with $2 a^{\dagger} a$ in Eqs. (6), we obtain the zero operators $a^{\dagger} a^{\dagger} a$ and $a^{\dagger} a a$ in the equation of motion for the occupation number $n_{a}(t)=a^{\dagger}(t) a(t)$. As follows from Eqs. (1), one should skip the terms $a^{\dagger} a^{\dagger} a$ and $a^{\dagger} a a$ in the equation of motion for $n_{a}(t)$ (see Appendix A). Because our aim is to derive and study $n_{a}(t)$, we disregard the terms proportional to $2 a^{\dagger} a$ in Eqs. (6) (Appendix A). Note that, for bosonic systems, the equations for the creation and annihilation operators coincide with Eqs. (6) without the terms proportional to $2 a^{\dagger} a$. The procedure for obtaining the occupation number of collective subsystem is well established $[23,41]$. The details related to the FC oscillator are given in Appendix B. Here, we directly write the final expression for the time dependence of occupation number for fermionic (f) and bosonic (b) collective subsystem:

$$
\begin{aligned}
n_{\mathrm{f}, \mathrm{b}}^{\mathrm{FC}}(t)= & A^{*}(t) A(t) n_{\mathrm{f}, \mathrm{b}}^{\mathrm{FC}}(0) \\
& +B^{*}(t) B(t)\left[1 \mp n_{\mathrm{f}, \mathrm{b}}^{\mathrm{FC}}(0)\right]+\mathrm{I}_{\mathrm{f}, \mathrm{b}}^{\mathrm{FC}}(t)+\mathrm{J}_{\mathrm{f}, \mathrm{b}}^{\mathrm{FC}}(t),
\end{aligned}
$$

where

$$
\mathrm{I}_{\mathrm{f}, \mathrm{b}}^{\mathrm{FC}}(t)=\frac{g_{0}}{\pi} \int_{0}^{\infty} d w \frac{\gamma^{2} w}{\gamma^{2}+w^{2}} n_{\mathrm{f}, \mathrm{b}}(w) M^{*}(w, t) M(w, t),
$$

and

$$
\mathrm{J}_{\mathrm{f}, \mathrm{b}}^{\mathrm{FC}}(t)=\frac{g_{0}}{\pi} \int_{0}^{\infty} d w \frac{\gamma^{2} w}{\gamma^{2}+w^{2}}\left[1 \mp n_{\mathrm{f}, \mathrm{b}}(w)\right] N^{*}(w, t) N(w, t) .
$$

The upper sign in Eqs. (7) and (9) corresponds to the fermionic subsystem with the $n_{\mathrm{f}}(w)=\{\exp [\hbar w /(k T)]+1\}^{-1}$ equilibrium Fermi-Dirac distribution, and the lower sign is related to bosonic subsystem (FC bosonic oscillator) with the $n_{\mathrm{b}}(w)=\{\exp [\hbar w /(k T)]-1\}^{-1}$ equilibrium BoseEinstein distribution. Here, an Ohmic spectral density with a Drude-Lorentz cutoff is used (see Appendix B). The similarity of expressions for the occupation numbers for fermionic and bosonic systems is the consequence of similarity of the equations of motion for creation and annihilation operators. The integrals in Eqs. (8) and (9) arise when we replace the sums over $v$ by integrals and introduce the bandwidth $\gamma$ of the bath. The memory time $\gamma^{-1}$ of the dissipation is the inverse of the bandwidth of the bath excitations which are coupled to the collective subsystem.

For the $A(t), B(t), M(w, t)$, and $N(w, t)$ the following expressions are obtained (see Appendix B):

$$
\begin{aligned}
A(t)= & i \frac{1}{2} \sum_{k=1}^{3} \beta_{k} e^{s_{k} t} \\
& \times\left\{\left(s_{k}-s_{0}\right)\left[\left(s_{k}+\gamma\right)\left(\Omega+\omega-2 i s_{k}\right)+2 g_{0} s_{k} \gamma\right]\right\}, \\
B(t)= & i \frac{1}{2} \sum_{k=1}^{3} \beta_{k} e^{s_{k} t} \\
& \times\left\{\left(s_{k}-s_{0}\right)\left[\left(s_{k}+\gamma\right)(\Omega-\omega)+2 g_{0} s_{k} \gamma\right]\right\}, \\
M(w, t)= & \left|\sum_{k=0}^{3} \beta_{k} e^{i s_{k} t}\left(s_{k}+\gamma\right)\left(s_{k}+i \omega\right)\right|^{2}, \\
N(w, t)= & \left|\sum_{k=0}^{3} \beta_{k} e^{i s_{k} t}\left(s_{k}+\gamma\right)\left(s_{k}-i \omega\right)\right|^{2},
\end{aligned}
$$

where $s_{0}=i w$ and $s_{1}, s_{2}$, and $s_{3}$ are the roots of the following equation:

$$
(s+\gamma)\left(s^{2}+\omega \Omega\right)+2 g_{0} \gamma \omega s=0,
$$

and

$$
\beta_{k}=\prod_{i=0}^{3} \frac{1}{\left(s_{k}-s_{i}\right)}, \quad k \neq i .
$$

In Eq. (11), $\Omega=\omega-2 g_{0} \gamma$ is the renormalized collective frequency which is the result of coupling between the collective and internal subsystems (see Appendix B).

As one of the main goals of our study is to elucidate the difference between FC and RWA oscillators, here we present also the expression for time dependence of the occupation number in the case of RWA coupling [41]:

$$
n_{\mathrm{f}, \mathrm{b}}^{\mathrm{RWA}}(t)=C^{*}(t) C(t) n_{\mathrm{f}, \mathrm{b}}^{\mathrm{RWA}}(0)+\mathrm{I}_{\mathrm{f}, \mathrm{b}}^{\mathrm{RWA}}(t),
$$

with

$$
\mathrm{I}_{\mathrm{f}, \mathrm{b}}^{\mathrm{RWA}}(t)=\frac{g_{0}}{\pi} \int_{0}^{\infty} d w \frac{\gamma^{2} w}{\gamma^{2}+w^{2}} n_{\mathrm{f}, \mathrm{b}}(w) L^{*}(w, t) L(w, t),
$$


where

$$
\begin{aligned}
C(t)= & e^{z_{1} t} \frac{z_{1}+\gamma}{z_{1}-z_{2}}+e^{z_{2} t} \frac{z_{2}+\gamma}{z_{2}-z_{1}}, \\
L(w, t)= & e^{z_{1} t} \frac{z_{1}+\gamma}{\left(z_{1}-z_{2}\right)\left(z_{1}-i w\right)} \\
& +e^{z_{2} t} \frac{z_{2}+\gamma}{\left(z_{2}-z_{1}\right)\left(z_{2}-i w\right)} \\
& +e^{i w t} \frac{i w+\gamma}{\left(i w-z_{1}\right)\left(i w-z_{2}\right)},
\end{aligned}
$$

and $z_{1}$ and $z_{2}$ are the roots of the equation

$$
(z+\gamma)(z-i \Omega)-i g_{0} \gamma z=0 .
$$

Note that, in Eq. (16), the renormalized frequency is $\Omega=$ $\omega-g_{0} \gamma$.

First, we note the structural difference of $n_{\mathrm{f}, \mathrm{b}}^{\mathrm{FC}}(t)$ and $n_{\mathrm{f}, \mathrm{b}}^{\mathrm{RWA}}(t)$. In the FC case there are two additional terms $B^{*}(t) B(t)[1 \mp$ $\left.n_{\mathrm{f}, \mathrm{b}}^{\mathrm{FC}}(0)\right]$ and $\mathrm{J}_{\mathrm{f}, \mathrm{b}}^{\mathrm{FC}}(t)$ which have no analogy in the RWA case. While in the RWA case the dynamics of occupation numbers of initially unoccupied states $\left[n_{\mathrm{f}, \mathrm{b}}^{\mathrm{RWA}}(0)=0\right]$ is determined by the integral term only, in the FC case the behavior of occupation number is determined by both integral and nonintegral terms [due to the presence of the $B^{*}(t) B(t)\left[1 \mp n_{\mathrm{fc} b}^{\mathrm{FC}}(0)\right]$ term]. As found, the contribution of the $B^{*}(t) B(t)\left[1 \mp n_{\mathrm{f}, \mathrm{b}}^{\mathrm{FC}}(0)\right]$ term to $n_{\mathrm{f}, \mathrm{b}}^{\mathrm{FC}}(t)$ is especially important in the short-time range.

The second difference between the FC and RWA oscillators is related to the difference between Eqs. (11) and (16) which determine the number of the roots. In FC coupling, the occupation numbers depend on three roots, while in the RWA case there are only two roots.

In the considered range of parameters $\gamma, \Omega$, and $g_{0}$, Eq. (11) has one negative real root $s_{1}$ and two complex-conjugated roots $s_{2}=s_{3}^{*}$ with negative real parts and $\operatorname{Re}\left[s_{1}\right]<\operatorname{Re}\left[s_{2}\right]$. This type of structure of the roots leads to exponential damping of time evolution of the functions $A^{*}(t) A(t)$ and $B^{*}(t) B(t)$ proportional to $e^{\left(s_{2}+s_{3}\right) t}$.

For the RWA oscillator, Eq. (16) has two complex roots $z_{1}$ and $z_{2}$ with negative real parts. In this case $C^{*}(t) C(t) \sim$ $e^{\left(z_{2}+z_{2}^{*}\right) t}$ where $z_{2}$ is the root with the maximal real part: $\operatorname{Re}\left[z_{1}\right]<\operatorname{Re}\left[z_{2}\right]$. In Fig. 1, we compare the dependence of $s_{2}+s_{3}=s_{2}+s_{2}^{*}$ from Eq. (11) with $z_{2}+z_{2}^{*}$ from Eq. (16) on parameter $\gamma$ for different coupling strengths $g_{0}$. In the RWA case, this dependence is rather weak for all $g_{0}$, while in the FC case the value of $s_{2}+s_{2}^{*}$ increases with $\gamma$. The dependence of $s_{2}+s_{2}^{*}$ on $\gamma$ becomes stronger with increasing $g_{0}$.

The behavior of the roots is reflected in time dependencies of $A^{*}(t) A(t), B^{*}(t) B(t)$, and $C^{*}(t) C(t)$ (Fig. 2). In the FC case, the functions $A^{*}(t) A(t)$ and $B^{*}(t) B(t)$ falloff stronger with time than the function $C^{*}(t) C(t)$ in the RWA case. This means that the initially occupied state $\left[n_{\mathrm{f}, \mathrm{bWA}}^{\mathrm{FC}}(0) \neq 0\right]$ more strongly influences the dynamics of occupation numbers in the case of the RWA oscillator.

In Fig. 3, we show the time dependencies of integral terms $\mathrm{I}_{\mathrm{f}, \mathrm{b}}^{\mathrm{FC}}(t), \mathrm{J}_{\mathrm{f}, \mathrm{b}}^{\mathrm{FC}}(t)$, and $\mathrm{I}_{\mathrm{f}, \mathrm{b}}^{\mathrm{RWA}}(t)$ in the expressions for occupation numbers of fermionic and bosonic collective subsystems. After some transient time the occupation numbers reach their equilibrium values. As expected, in the case of the FC oscillator we get a stronger damping of oscillations and correspondingly reach the asymptotic values more rapidly for both fermions

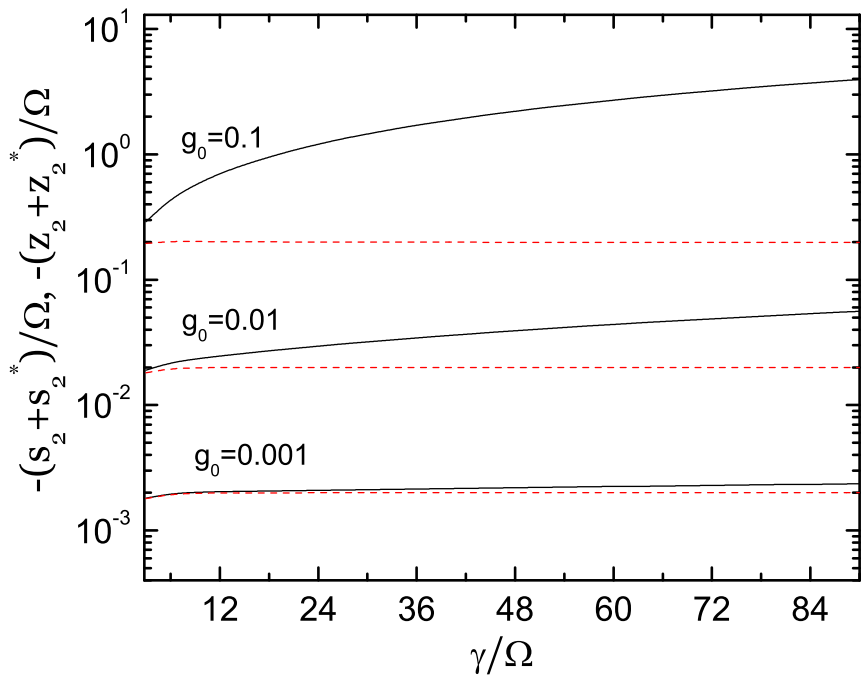

FIG. 1. The dependence of $s_{2}+s_{2}^{*}$ and $z_{2}+z_{2}^{*}$ on the parameter $\gamma$ for the indicated coupling constant $g_{0}$. The results of calculations for FC and RWA couplings are presented by solid and dashed lines, respectively.

and bosons. It is interesting to mention that $\mathrm{J}_{\mathrm{f}}^{\mathrm{FC}}(t)$ and $\mathrm{J}_{\mathrm{b}}^{\mathrm{FC}}(t)$ are almost the same. Moreover, at the values of $g_{0}$ and $T$ considered, these integral terms mainly contribute to $n_{\mathrm{f}, \mathrm{b}}^{\mathrm{FC}}(t)$. The role of $\mathrm{J}_{\mathrm{f}}^{\mathrm{FC}}(t)$ and $\mathrm{J}_{\mathrm{b}}^{\mathrm{FC}}(t)$ becomes smaller with increasing
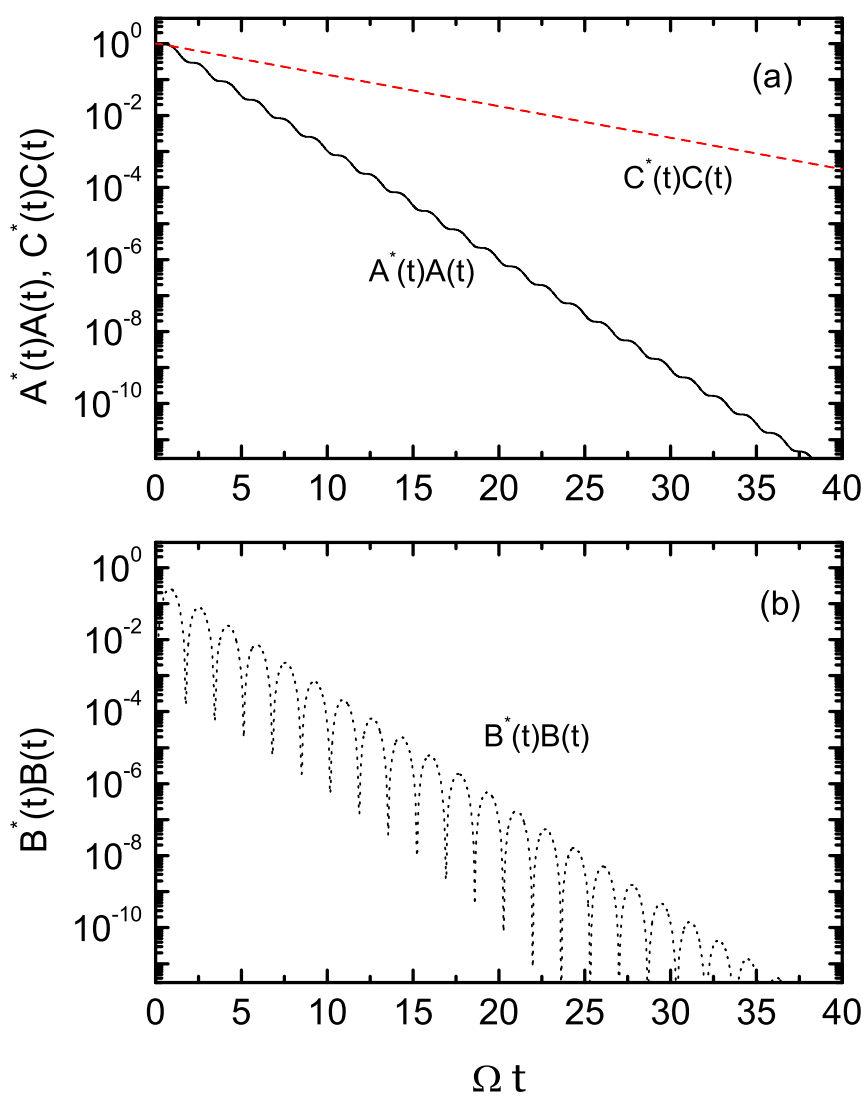

FIG. 2. The time dependence of indicated functions at $g_{0}=0.1$, $\gamma / \Omega=12$. 

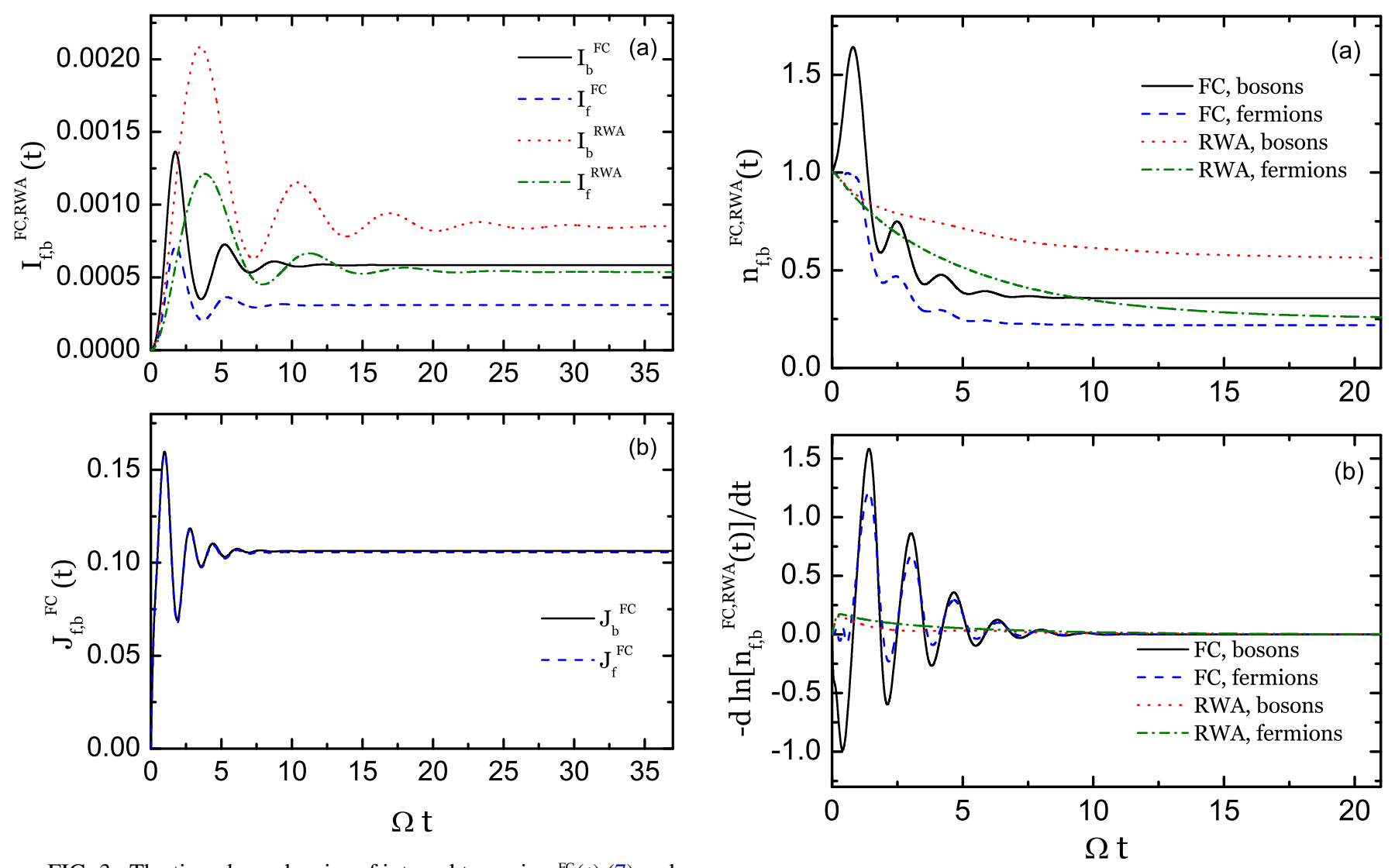

FIG. 3. The time dependencies of integral terms in $n_{\mathrm{f}, \mathrm{b}}^{\mathrm{FC}}(t)(7)$ and $n_{\mathrm{f}, \mathrm{b}}^{\mathrm{RWA}}(t)(13)$. The calculations are performed at $g_{0}=0.1, \gamma / \Omega=12$, and $k T /(\hbar \Omega)=0.1$.

temperature or with decreasing $g_{0}$. The transient time is almost independent of the statistical nature of the bath.

In Fig. 4, we show the time dependencies of occupation number and the value of $-\left(d n_{\mathrm{f}, \mathrm{b}}^{\mathrm{FC}} / d t\right) / n_{\mathrm{f}, \mathrm{b}}^{\mathrm{FC}}$ of the initially occupied state $n_{\mathrm{f}, \mathrm{b}}^{\mathrm{FC}, \mathrm{RWA}}(0)=1$. The occupation numbers $n_{\mathrm{f}, \mathrm{b}}^{\mathrm{FC}}(t)$ reach their equilibrium values faster then $n_{\mathrm{f}, \mathrm{b}}^{\mathrm{RWA}}(t)$ for both fermionic and bosonic collective subsystems. However, the oscillation before reaching the asymptotes are stronger in the case of a FC oscillator. So, the time behavior of occupation numbers is mostly determined by the kind of coupling rather than the nature of the system.

\section{DIFFERENTIAL EQUATION FOR OCCUPATION NUMBER}

By using the explicit time dependence of $n_{\mathrm{f}, \mathrm{b}}^{\mathrm{FC}}(t)$ [Eq. (7)], we derive the following differential equation:

$$
\frac{d}{d t} n_{\mathrm{f}, \mathrm{b}}^{\mathrm{FC}}(t)=-2 \lambda_{\mathrm{f}, \mathrm{b}}^{\mathrm{FC}}(t) n_{\mathrm{f}, \mathrm{b}}^{\mathrm{FC}}(t)+2 D_{\mathrm{f}, \mathrm{b}}^{\mathrm{FC}}(t),
$$

where

$$
\lambda_{\mathrm{f}, \mathrm{b}}^{\mathrm{FC}}(t)=-\frac{1}{2} \frac{d \ln \left[A^{*}(t) A(t) \mp B^{*}(t) B(t)\right]}{d t},
$$

and

$$
\begin{aligned}
D_{\mathrm{f}, \mathrm{b}}^{\mathrm{FC}}(t)= & \lambda_{\mathrm{f}, \mathrm{b}}^{\mathrm{FC}}(t)\left[B^{*}(t) B(t)+\mathrm{I}_{\mathrm{f}, \mathrm{b}}^{\mathrm{FC}}(t)+\mathrm{J}_{\mathrm{f}, \mathrm{b}}^{\mathrm{FC}}(t)\right] \\
& +\frac{1}{2} \frac{d}{d t}\left[B^{*}(t) B(t)+\mathrm{I}_{\mathrm{f}, \mathrm{b}}^{\mathrm{FC}}(t)+\mathrm{J}_{\mathrm{f}, \mathrm{b}}^{\mathrm{FC}}(t)\right]
\end{aligned}
$$

In Eq. (18) the upper (lower) sign corresponds to fermionic (bosonic) subsystems.

The same type differential equation was obtain for the RWA oscillator [41], where

$$
\lambda_{\mathrm{f}, \mathrm{b}}^{\mathrm{RWA}}(t)=-\frac{1}{2} \frac{d \ln \left[C^{*}(t) C(t)\right]}{d t},
$$

and

$$
D_{\mathrm{f}, \mathrm{b}}^{\mathrm{RWA}}(t)=\lambda_{\mathrm{f}, \mathrm{b}}^{\mathrm{RWA}}(t) \mathrm{I}_{\mathrm{f}, \mathrm{b}}^{\mathrm{RWA}}(t)+\frac{d}{d t} \mathrm{I}_{\mathrm{f}, \mathrm{b}}^{\mathrm{RWA}}(t)
$$

are the time-dependent coefficients.

\section{Comparison with discretized environment method}

As a cross-check of the analytical solutions given above, we also applied the discretized environment method [28] generalized to treat the FC case. The simple idea developed in this method is that the continuous environment can be accurately discretized so that the equations of motion are solved by a direct diagonalization of the complete (collective subsystem + environment) Hamiltonian. Starting from the 
equation of motion (6) and using the same approximation as in previous section, the evolution can be rewritten as

$$
i \hbar \frac{d}{d t}\left(\begin{array}{c}
\mathbf{C} \\
\mathbf{C}^{\dagger}
\end{array}\right)=\mathbf{L}\left(\begin{array}{c}
\mathbf{C} \\
\mathbf{C}^{\dagger}
\end{array}\right),
$$

where $\mathbf{C}^{t}=\left(a,\left\{a_{\nu}\right\}_{\nu=1, N}\right)$ contains the annihilation operators of the collective subsystem and bath. $N$ is the number of states used to discretize the environment. The discretization procedure is described in Ref. [28] and we only give below novel aspects associated with the FC case compared with the RWA case. $\mathbf{L}$ is a $4 \times 4$ block matrix with

$$
\mathbf{L}=\left(\begin{array}{cc}
L^{--} & L^{-+} \\
-L^{-+} & L^{++}
\end{array}\right),
$$

where each matrix is of size $(N+1) \times(N+1)$. Using the convention that $v=0$ labels the operator associated with the collective subsystem, we have

$$
\begin{aligned}
& L_{00}^{--}=\hbar \omega, \quad L_{v v}^{--}=\hbar \omega_{\nu}, \quad L_{0 v}^{--}=L_{\nu 0}^{--}=g_{v}, \\
& L_{00}^{++}=-\hbar \omega, \quad L_{v v}^{--}=-\hbar \omega_{v}, \\
& L_{0 v}^{++}=L_{\nu 0}^{++}=-g_{v}, \quad L_{0 v}^{-+}=L_{\nu 0}^{-+}=+g_{v},
\end{aligned}
$$

while other matrix elements are zero. The matrix $\mathbf{L}$ corresponds to the generalized Hamiltonian found for superfluid systems. It could be diagonalized by using a standard Bogoliubov transformation [42]. The RWA case is recovered simply if $L^{-+}=0$. The diagonalization gives new operators given by

$$
\begin{aligned}
& A_{\alpha}=\sum_{\lambda} U_{\lambda}^{\alpha} C_{\lambda}-V_{\lambda}^{\alpha} C_{\lambda}^{\dagger}, \\
& A_{\alpha}^{\dagger}=\sum_{\lambda} U_{\lambda}^{\alpha} C_{\lambda}^{\dagger}-V_{\lambda}^{\alpha} C_{\lambda},
\end{aligned}
$$

where $U$ and $V$ are the usual Bogoliubov matrices. The new operators evolve through

$$
A_{\alpha}^{\dagger}(t)=e^{i \Omega_{\alpha} t} A_{\alpha}^{\dagger}(0)
$$

where $\Omega_{\alpha}$ are the eigenvalues of $\mathbf{L}$. Using this representation, as in Ref. [28], one can easily obtain the expectation values of the occupation probability for the environment. An illustration of the results of the discretized environment method is given in Fig. 5 and compared with the Langevin-approach result. The alternative direct solution provided by the discretized environment method validates the different analytical results given in this paper. Below, we only show the quantum Langevin-approach result.

\section{ASYMPTOTIC OCCUPATION NUMBERS}

Because the roots of Eq. (11) have negative real parts, $A^{*}(t \rightarrow \infty) A(t \rightarrow \infty)=0, B^{*}(t \rightarrow \infty) B(t \rightarrow \infty)=0$ and the asymptotic occupation numbers are defined only by the integral terms:

$$
n_{\mathrm{f}, \mathrm{b}}^{\mathrm{FC}}(t \rightarrow \infty)=\mathrm{I}_{\mathrm{f}, \mathrm{b}}^{\mathrm{FC}}(t \rightarrow \infty)+\mathrm{J}_{\mathrm{f}, \mathrm{b}}^{\mathrm{FC}}(t \rightarrow \infty) .
$$
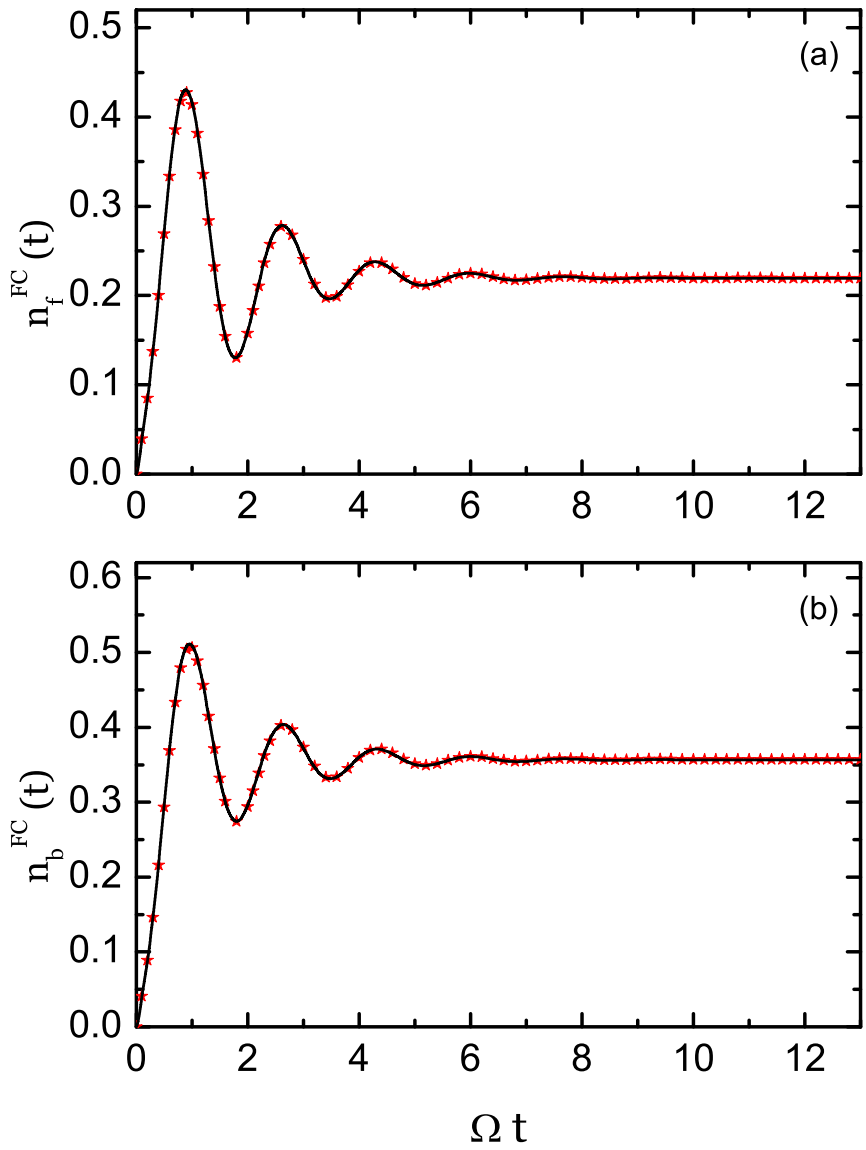

FIG. 5. Time evolution of (a) $n_{\mathrm{f}}^{\mathrm{FC}}(t)$ and (b) $n_{\mathrm{b}}^{\mathrm{FC}}(t)$ obtained with the discretized environment method applied to the FC case (star symbols) and compared with the results of the quantum Langevin approach (solid lines) for $n_{\mathrm{f}, \mathrm{b}}^{\mathrm{FC}}(0)=0, g_{0}=0.1, \gamma / \Omega=12$, and $k T /(\hbar \Omega)=1$. The results of the two approaches are almost identical.

The following expressions are found for the asymptotes of these integral terms:

$$
\begin{aligned}
\mathrm{I}_{\mathrm{f}, \mathrm{b}}^{\mathrm{FC}}(t \rightarrow \infty)= & \frac{g_{0}}{\pi} \int_{0}^{\infty} d w n_{\mathrm{f}, \mathrm{b}}(w) \\
& \times \frac{\gamma^{2}}{\gamma^{2}+w^{2}} \frac{w\left(w^{2}+\gamma^{2}\right)(w+\omega)^{2}}{\left(w^{2}+s_{1}^{2}\right)\left(w^{2}+s_{2}^{2}\right)\left(w^{2}+s_{3}^{2}\right)},
\end{aligned}
$$

and

$$
\begin{aligned}
\mathrm{J}_{\mathrm{f}, \mathrm{b}}^{\mathrm{FC}}(t \rightarrow \infty)= & \frac{g_{0}}{\pi} \int_{0}^{\infty} d w\left[1 \mp n_{\mathrm{f}, \mathrm{b}}(w)\right] \\
& \times \frac{\gamma^{2}}{\gamma^{2}+w^{2}} \frac{w\left(w^{2}+\gamma^{2}\right)(w-\omega)^{2}}{\left(w^{2}+s_{1}^{2}\right)\left(w^{2}+s_{2}^{2}\right)\left(w^{2}+s_{3}^{2}\right)} .
\end{aligned}
$$

For the FC fermionic oscillator, one can rewrite Eq. (28) as

$$
n_{\mathrm{f}}^{\mathrm{FC}}(t \rightarrow \infty)=\mathrm{I}_{\mathrm{f}}^{T}+\mathrm{I}^{C}
$$



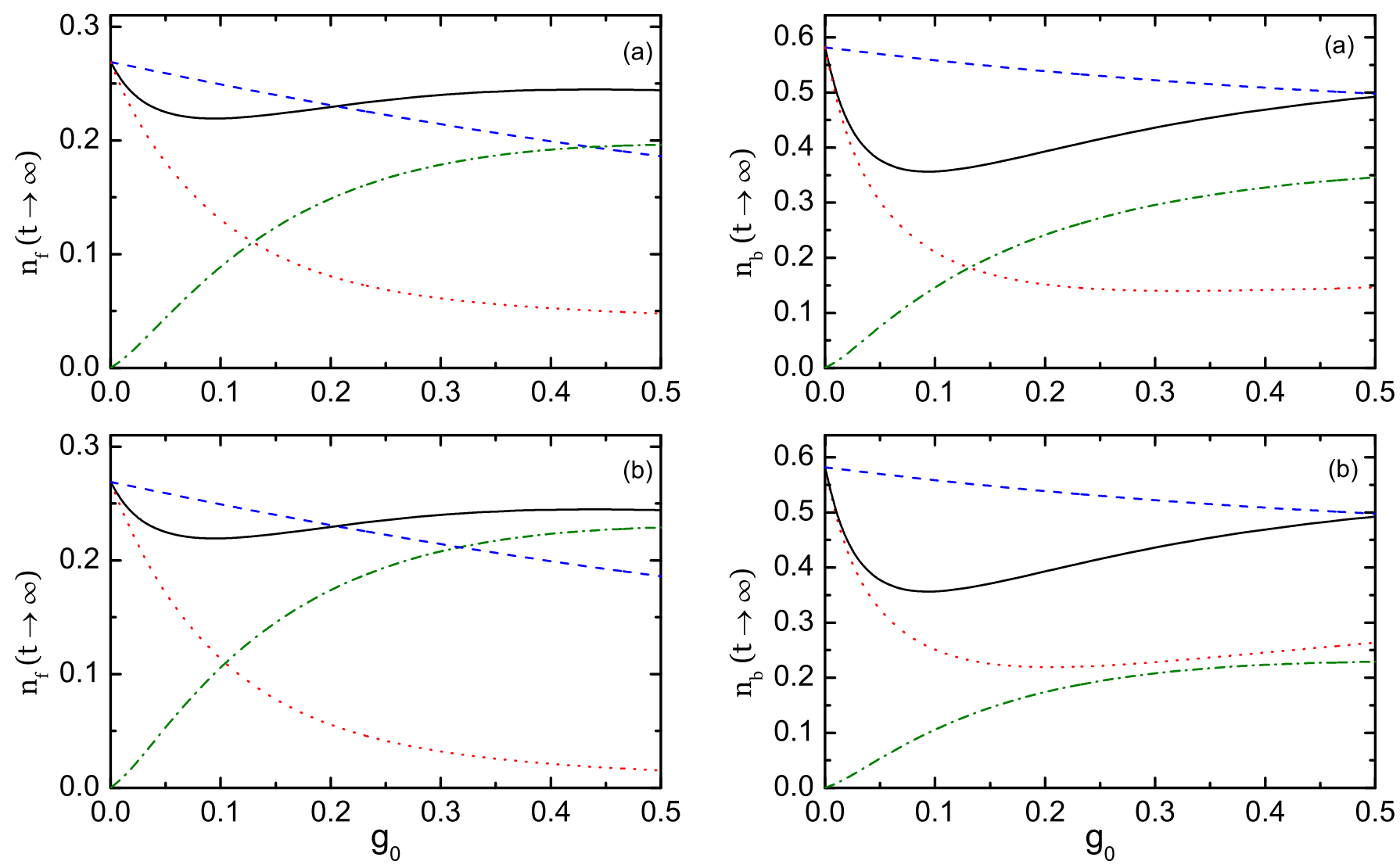

FIG. 6. The dependencies of asymptotic occupation numbers $n_{\mathrm{f}}(t \rightarrow \infty)$ on the coupling strength $g_{0}$ for the FC (solid lines) and RWA (dashed lines) fermionic oscillators. (a) The separate contributions of $\mathrm{I}_{\mathrm{f}}^{\mathrm{FC}}(t \rightarrow \infty)$ and $\mathrm{J}_{\mathrm{f}}^{\mathrm{FC}}(t \rightarrow \infty)$ in Eq. (28) are presented by dotted and dash-dotted lines, respectively. (b) The separate contributions of $\mathrm{I}_{\mathrm{f}}^{T}$ and $\mathrm{I}^{C}$ in Eq. (31) are presented by dotted and dash-dotted lines, respectively. The calculations are performed at $\gamma / \Omega=12$, and $k T /(\hbar \Omega)=1$.

to distinguish the term explicitly depending on temperature,

$$
\begin{aligned}
\mathbf{I}_{\mathrm{f}}^{T}= & \frac{g_{0}}{\pi} \int_{0}^{\infty} d w n_{\mathrm{f}}(w) \\
& \times \frac{\gamma^{2}}{\gamma^{2}+w^{2}} \frac{4 \omega w^{2}\left(w^{2}+\gamma^{2}\right)}{\left(w^{2}+s_{1}^{2}\right)\left(w^{2}+s_{2}^{2}\right)\left(w^{2}+s_{3}^{2}\right)},
\end{aligned}
$$

and the term independent of temperature,

$$
\begin{aligned}
\mathrm{I}^{c}= & \frac{g_{0} \gamma^{2}}{\pi} \frac{\left(s_{1}^{2}-\omega^{2}\right) \ln \left(s_{1}^{2}\right)}{2\left(s_{1}^{2}-s_{2}^{2}\right)\left(s_{1}^{2}-s_{3}^{2}\right)} \\
& +\frac{g_{0} \gamma^{2}}{\pi}\left[\frac{\left(s_{2}^{2}-\omega^{2}\right) \ln \left(s_{2}^{2}\right)}{2\left(s_{2}^{2}-s_{1}^{2}\right)\left(s_{2}^{2}-s_{3}^{2}\right)}+\frac{\left(s_{3}^{2}-\omega^{2}\right) \ln \left(s_{3}^{2}\right)}{2\left(s_{3}^{2}-s_{1}^{2}\right)\left(s_{3}^{2}-s_{2}^{2}\right)}\right] \\
& +g_{0} \gamma^{2} \omega \frac{\left(s_{1}-s_{2}\right)\left(s_{2}-s_{3}\right)\left(s_{3}-s_{1}\right)}{2\left(s_{1}^{2}-s_{2}^{2}\right)\left(s_{2}^{2}-s_{3}^{2}\right)\left(s_{3}^{2}-s_{1}^{2}\right)} .
\end{aligned}
$$

By analogy the expression for the FC bosonic oscillator reads

$$
n_{\mathrm{b}}^{\mathrm{FC}}(t \rightarrow \infty)=\mathrm{I}_{\mathrm{b}}^{T}+\mathrm{I}^{C},
$$

FIG. 7. The same as in Fig. 6, but for the FC and RWA bosonic oscillators.

where

$$
\begin{aligned}
\mathbf{I}_{\mathrm{b}}^{T}= & \frac{g_{0}}{\pi} \int_{0}^{\infty} d w n_{\mathrm{b}}(w) \\
& \times \frac{\gamma^{2}}{\gamma^{2}+w^{2}} \frac{2 w\left(w^{2}+\gamma^{2}\right)\left(w^{2}+\omega^{2}\right)}{\left(w^{2}+s_{1}^{2}\right)\left(w^{2}+s_{2}^{2}\right)\left(w^{2}+s_{3}^{2}\right)},
\end{aligned}
$$

and the term $\mathrm{I}^{C}$ is the same as in Eq. (31). These expressions are used further to study the asymptotic behavior of occupation numbers.

In Figs. 6 and 7, the dependencies of asymptotic occupation numbers $n_{\mathrm{f}, \mathrm{b}}(t \rightarrow \infty)$ on the coupling strength $g_{0}$ are shown for fermionic and bosonic collective subsystems and for both types of couplings. In the case of RWA coupling (dashed lines), the values of $n_{\mathrm{f}, \mathrm{b}}^{\mathrm{RWA}}(t \rightarrow \infty)$ monotonically decrease with increasing $g_{0}$ for both fermionic and bosonic collective subsystem. The dependence of $n_{\mathrm{f}, \mathrm{b}}^{\mathrm{FC}}(t \rightarrow \infty)$ on $g_{0}$ is more complicated. For the fermionic collective subsystem, the value of $n_{\mathrm{f}}^{\mathrm{FC}}(t \rightarrow \infty)$ falls down with increasing $g_{0}$ up to $g_{0} \approx$ 0.1 and then starts to increase up to $g_{0} \approx 0.45$ reaching some maximal value. The further increase of $g_{0}$ causes a decrease of $n_{\mathrm{f}}^{\mathrm{FC}}(t \rightarrow \infty)$. Such a behavior of occupation number is due to the contributions of two terms, i.e., $\mathrm{I}_{\mathrm{f}}^{\mathrm{FC}}(t \rightarrow \infty)$ [dotted line in Fig. 6(a)] and $\mathbf{J}_{\mathrm{f}}^{\mathrm{FC}}(t \rightarrow \infty)$ [dash-dotted line in Fig. 6(a)], which have different dependencies on $g_{0}$. As seen in Fig. 6(b), the temperature-dependent term (dotted line) decreases with increasing $g_{0}$ while the term $\mathrm{I}_{\mathrm{f}}^{C}$ (dash-dotted line) increases. So, the contributions of increasing and decreasing terms provide the complicated dependence of asymptotic occupation number on coupling strength. Note that the range of $g_{0}$ considered includes both weak- and strong-coupling regimes. 
Very similar results we have for bosonic collective system (Fig. 7). However, the dependence of temperature part of $\mathrm{I}_{\mathrm{b}}^{T}$ on $g_{0}$ [dotted line in Fig. 7(b)] differs from its fermionic analogous. For bosonic system, the value of $\mathrm{I}_{\mathrm{b}}^{T}$ decreases with increasing $g_{0}$ up to $g_{0} \approx 0.2$, and further increase of $g_{0}$ leads to increasing $\mathrm{I}_{\mathrm{b}}^{T}$.

\section{WEAK-COUPLING LIMIT}

Quite simple expressions for the occupation numbers are obtained in the weak-coupling limit, $g_{0} \ll 1$. Retaining the terms to leading order in $g_{0}$, we obtain for the roots of equation (11) the following expressions:

$$
\begin{aligned}
& s_{1}=-\gamma+2 g_{0} \omega, \\
& s_{2}=-g_{0} \omega-i \omega, \\
& s_{3}=-g_{0} \omega+i \omega .
\end{aligned}
$$

With these roots Eq. (33) reads

$$
\mathrm{I}^{c}=\frac{g_{0}}{\pi} \frac{\gamma^{2}\left(-\gamma^{2}+\pi \gamma \omega-\omega^{2}+\gamma^{2} \ln \left[\frac{\gamma}{\omega}\right]-\omega^{2} \ln \left[\frac{\gamma}{\omega}\right]\right)}{\left(\gamma^{2}+\omega^{2}\right)^{2}} .
$$

Assuming $\omega \ll \gamma$, we get

$$
\mathrm{I}^{c} \approx g_{0} \frac{1}{\pi} \ln \left[\frac{\gamma}{\omega}\right]
$$

Replacing the roots in Eqs. (32) and (35) by those from Eqs. (36), the temperature terms of asymptotic occupation numbers for fermionic and bosonic systems are

$$
\mathbf{I}_{\mathrm{f}}^{T}=\frac{1}{\pi} g_{0} \gamma^{2} \int_{0}^{\infty} d w \frac{1}{\exp \left[\frac{\hbar w}{k T}\right]+1} \frac{4 w^{2} \omega}{\left[w^{4}+2\left(-1+g_{0}^{2}\right) w^{2} \omega^{2}+\left(1+g_{0}^{2}\right)^{2} \omega^{4}\right]\left[w^{2}+\left(\gamma-2 g_{0} \omega\right)^{2}\right]}
$$

and

$$
\mathbf{I}_{\mathrm{b}}^{T}=\frac{1}{\pi} g_{0} \gamma^{2} \int_{0}^{\infty} d w \frac{1}{\exp \left[\frac{\hbar w}{k T}\right]-1} \frac{2 w\left(w^{2}+\omega^{2}\right)}{\left[w^{4}+2\left(-1+g_{0}^{2}\right) w^{2} \omega^{2}+\left(1+g_{0}^{2}\right)^{2} \omega^{4}\right]\left[w^{2}+\left(\gamma-2 g_{0} \omega\right)^{2}\right]}
$$

The integrands in Eqs. (39) and (40) are the $\delta$-type functions at $g_{0} \rightarrow 0$. At high temperatures $(k T \gg \hbar \omega)$ the main contributions to the integrals (39) and (40) arise from the neighborhood of $|w| \approx \omega$ and yield the usual Fermi-Dirac and Bose-Einstein expressions

$$
\begin{aligned}
\mathrm{I}_{\mathrm{f}}^{T} & =\frac{1}{\exp \left[\frac{\hbar \omega}{k T}\right]+1}, \\
\mathrm{I}_{\mathrm{b}}^{T} & =\frac{1}{\exp \left[\frac{\hbar \omega}{k T}\right]-1} .
\end{aligned}
$$

This means that the bath imposes its temperature to the collective subsystem. In the high-temperature limit we recover a Markovian dynamics [1,2].

At $\gamma \rightarrow \infty$ and $g_{0} \ll 1$ (Markovian weak-coupling limit) we obtain

$$
\mathbf{I}_{\mathrm{f}}^{T}=\frac{g_{0}}{\pi} \int_{0}^{\infty} d w \frac{1}{\exp \left[\frac{\hbar w}{k T}\right]+1} \frac{4 w^{2} \omega}{w^{4}-2 w^{2} \omega^{2}+\omega^{4}},
$$

and

$$
\mathbf{I}_{\mathrm{b}}^{T}=\frac{g_{0}}{\pi} \int_{0}^{\infty} d w \frac{1}{\exp \left[\frac{\hbar w}{k T}\right]-1} \frac{2 w\left(w^{2}+\omega^{2}\right)}{w^{4}-2 w^{2} \omega^{2}+\omega^{4}} .
$$

At the low-temperature limit $(k T \ll \hbar \omega)$, in Eqs. (42) and (43) the expansion

$$
\frac{1}{\left(w^{4}-2 w^{2} \omega^{2}+\omega^{4}\right)} \approx \frac{1}{\omega^{4}}\left[1+2\left(\frac{w}{\omega}\right)^{2}\right]
$$

can be employed to obtain the following analytical expressions:

$$
\mathbf{I}_{\mathrm{f}}^{T}=\frac{6 g_{0}}{\pi}\left(\frac{k T}{\hbar \omega}\right)^{2}\left[\left(\frac{k T}{\hbar \omega}\right) \zeta(3)+30\left(\frac{k T}{\hbar \omega}\right)^{3} \zeta(5)\right]
$$

and

$$
\mathrm{I}_{\mathrm{b}}^{T}=\frac{2 g_{0}}{\pi}\left(\frac{k T}{\hbar \omega}\right)^{2}\left[\zeta(2)+18\left(\frac{k T}{\hbar \omega}\right)^{2} \zeta(4)\right],
$$

for fermionic and bosonic systems, respectively. Here, $\zeta(n)$ is the Riemann zeta function. Note that, in the case of a RWA oscillator we obtained in Ref. [41]

$$
\mathrm{I}_{\mathrm{f}}^{\mathrm{RWA}}=\frac{g_{0}}{2 \pi}\left(\frac{k T}{\hbar \Omega}\right)^{2}\left[\zeta(2)+6 \frac{k T}{\hbar \Omega} \zeta(3)\right]
$$

for fermionic subsystem and

$$
\mathrm{I}_{\mathrm{b}}^{\mathrm{RWA}}=\frac{g_{0}}{\pi}\left(\frac{k T}{\hbar \Omega}\right)^{2}\left[\zeta(2)+4 \frac{k T}{\hbar \Omega} \zeta(3)\right]
$$

for the bosonic subsystem. While in the case of a RWA oscillator the ratio $\mathrm{I}_{\mathrm{f}}^{\mathrm{RWA}} / \mathrm{I}_{\mathrm{b}}^{\mathrm{RWA}} \approx 1 / 2$, in the case of FC oscillator the ratio $\mathrm{I}_{\mathrm{f}}^{T} / \mathrm{I}_{\mathrm{b}}^{T}$ depends on $T$.

In Fig. 8, the temperature dependencies of asymptotic values of $\mathrm{I}_{\mathrm{f}, \mathrm{b}}^{T}$ (solid lines) are shown for fermionic and bosonic collective subsystems. The calculations are performed at $g_{0}=0.001, \gamma / \Omega=12$. For fermionic (bosonic) system at temperatures $k T /(\hbar \Omega) \geqslant 0.3, \mathrm{I}_{\mathrm{f}}^{T}\left(\mathrm{I}_{\mathrm{b}}^{T}\right)$ almost coincides with the usual Fermi-Dirac (Bose-Einstein) distribution. At low temperatures $k T /(\hbar \Omega) \leqslant 0.04$ the expressions (45) and (46) seem to be a very good approximation. In Fig. 8, we also mark the value of a temperature-independent part $\mathrm{I}^{c}=5.7 \times 10^{-4}$ of asymptotic occupation numbers. As seen, this term has a valuable contribution to the occupation number only at small temperature. Because at $g_{0} \rightarrow 0$ we get $\mathrm{I}^{c} \rightarrow 0$ (see Figs. 6 and 7), the part $\mathrm{I}^{c}$ plays a major role in asymptotic occupation numbers at small temperature and relatively large $g_{0}$. 

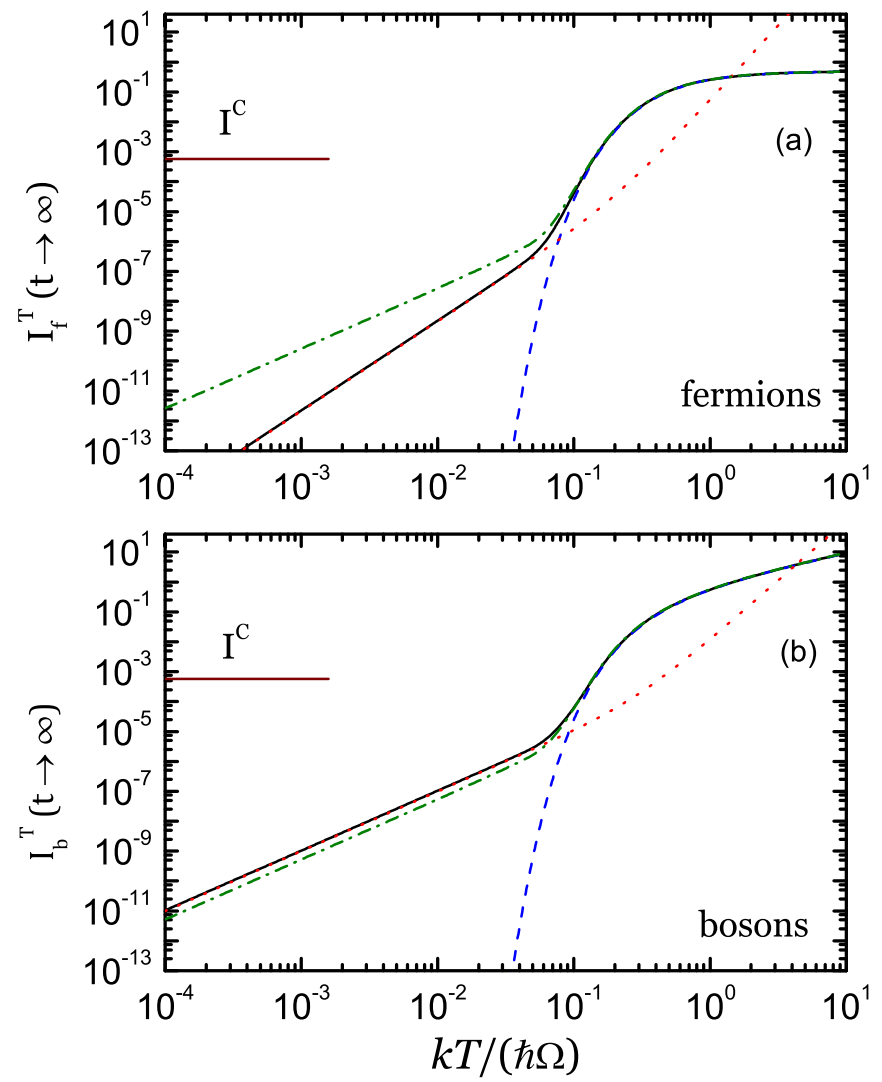

FIG. 8. The calculated dependencies of temperature parts $I_{\mathrm{f}, \mathrm{b}}^{T}$ of the asymptotic occupation numbers for (a) a FC fermionic oscillator and (b) for a FC bosonic oscillator at temperature $T$ (solid lines). The occupation numbers for the Fermi-Dirac and Bose-Einstein distributions are presented by dashed lines. The leading-order expressions obtained for fermions and bosons in power of $(k T) /(\hbar \omega)$, Eqs. (45) and (46), are presented by dotted lines. The calculated dependencies of asymptotic $\mathrm{I}_{\mathrm{f}, \mathrm{b}}^{\mathrm{RWA}}$ in the case of a RWA oscillator are presented by dash-dotted lines. The value of $\mathrm{I}^{C}=5.7 \times 10^{-4}$ is marked by the horizontal line. The calculations are performed at $g_{0}=0.001$ and $\gamma / \Omega=12$.

\section{SUMMARY}

The non-Markovian quantum Langevin equations were derived for FC fermionic and bosonic oscillators. The explicit expressions for the time-dependent occupation numbers were obtained. The initially occupied state influences the dynamics stronger in the case of a RWA oscillator rather than in the case of a FC oscillator, i.e., the decay of occupied states is slower in this case than in the case of a FC oscillator. At the initial time, the time-dependence of the occupation number oscillates more in the case of a FC oscillator. While the asymptotic occupation numbers in the RWA oscillator are defined only the temperature-dependent term, the temperature-independent term contributes to the asymptotic occupation numbers in FC oscillators as well. The former term becomes important at low temperature and relatively large coupling strength. In FC oscillator, the contributions of these two terms provide a more complicated dependence of asymptotic occupation numbers on the coupling strength than in the case of a RWA oscillator. The transient time of occupation numbers is almost independent of the statistical nature of the bath. The results of numerical calculations of occupation numbers were found to be identical to those obtained with the discretized environment method.

\section{ACKNOWLEDGMENTS}

V.V.S. is thankful to the Mechnikov Program (France) for partial support. G.G.A. and N.V.A. acknowledge partial support from the Alexander von Humboldt-Stiftung (Bonn). The IN2P3(France)-JINR(Dubna) Cooperation Program is gratefully acknowledged. This work was supported by RFBR (Moscow) and DFG (Bonn).

\section{APPENDIX A}

Using the general equations of motion for the creation $a^{\dagger}(t)$ and annihilation $a(t)$ operators (6) and the commutation relations $\left(a^{\dagger}\right)^{2}=a^{2}=0$, we obtain the equation

$$
\begin{aligned}
\frac{d n_{a}(t)}{d t}= & \frac{i}{\hbar} \sum_{v} g_{v}\left[a(t)-a^{\dagger}(t)\right]\left[a_{v}^{\dagger}(t)-a_{v}(t)\right] \\
= & \frac{i}{\hbar} \sum_{v} g_{v}\left[a_{v}^{\dagger}(t) a(t)-a^{\dagger}(t) a_{v}(t)\right. \\
& \left.+a(t) a_{v}(t)-a^{\dagger}(t) a_{v}^{\dagger}(t)\right]
\end{aligned}
$$

for the occupation number $n_{a}(t)=a^{\dagger}(t) a(t)$ of the collective subsystem. For the operators $a_{v}^{\dagger}(t) a(t)-a^{\dagger}(t) a_{v}(t)$ and $a(t) a_{v}(t)-a^{\dagger}(t) a_{v}^{\dagger}(t)$ in Eq. (A1), one can derive the following equations:

$$
\begin{aligned}
\frac{d^{2}}{d t^{2}}\left(a_{v}^{\dagger} a-a^{\dagger} a_{v}\right)= & -\left[\omega-\omega_{v}\right]^{2}\left(a_{v}^{\dagger} a-a^{\dagger} a_{v}\right) \\
& +\frac{2 i g_{v}}{\hbar} \frac{d}{d t}\left(n_{a}-n_{a_{v}}\right), \\
\frac{d^{2}}{d t^{2}}\left(a a_{v}-a^{\dagger} a_{v}^{\dagger}\right)= & -\left[\omega+\omega_{v}\right]^{2}\left(a a_{v}-a^{\dagger} a_{v}^{\dagger}\right) \\
& +\frac{2 i g_{v}}{\hbar} \frac{d}{d t}\left(n_{a}+n_{a_{v}}-1\right),
\end{aligned}
$$

where $n_{a_{v}}(t)=a_{v}^{\dagger}(t) a_{v}(t)$ are the occupation numbers of the bosonic subsystem. To obtain Eqs. (A2), we employ the random-phase approximation for the heat-bath degrees of freedom:

$$
\begin{aligned}
& \sum_{v^{\prime}} g_{v^{\prime}}\left(a_{v^{\prime}}^{\dagger} a_{v}+a_{v}^{\dagger} a_{v^{\prime}}\right) \simeq 2 g_{v} a_{v}^{\dagger} a_{v}, \\
& \sum_{v^{\prime}} g_{v^{\prime}}\left(a_{v^{\prime}}^{\dagger} a_{v}-a_{v}^{\dagger} a_{v^{\prime}}\right) \simeq 0 .
\end{aligned}
$$

Substituting the formal solutions of Eqs. (A2) into Eq. (A1) and taking $a(0) a_{v}(0)=0, a^{\dagger}(0) a_{v}^{\dagger}(0)=0, a_{v}^{\dagger}(0) a(0)=0$, and $a^{\dagger}(0) a_{v}(0)=0$, we rewrite Eq. (A1) as

$$
\begin{aligned}
\frac{d n_{a}(t)}{d t}= & \sum_{v} \int_{0}^{t} d s\left\{\mathcal{W}_{v}^{-}(t-s)\left[n_{a_{v}}(s)-n_{a}(s)\right]\right. \\
& \left.+\mathcal{W}_{v}^{\dagger}(t-s)\left[1-n_{a}(s)-n_{a_{v}}(s)\right]\right\},
\end{aligned}
$$


where

$$
\begin{aligned}
& \mathcal{W}_{v}^{-}=\frac{2 g_{v}^{2}}{\hbar^{2}} \cos \left(\left[\omega-\omega_{\nu}\right][t-s]\right), \\
& \mathcal{W}_{\nu}^{\dagger}=\frac{2 g_{v}^{2}}{\hbar^{2}} \cos \left(\left[\omega+\omega_{\nu}\right][t-s]\right) .
\end{aligned}
$$

This master equation is complemented by the set of master equations for $n_{a_{v}}$ :

$$
\begin{aligned}
\frac{d n_{a_{v}}(t)}{d t}= & \int_{0}^{t} d s\left\{\mathcal{W}_{v}^{-}(t-s)\left[n_{a}(s)-n_{a_{v}}(s)\right]\right. \\
& \left.-\mathcal{W}_{v}^{\dagger}(t-s)\left[1-n_{a}(s)-n_{a_{v}}(s)\right]\right\}
\end{aligned}
$$

In the case of RWA coupling, $\mathcal{W}_{v}^{\dagger}=0$ in Eqs. (A3) and (A4).
Note that the terms with $2 a^{\dagger} a$ in Eqs. (6) do not give any contribution to the equation of motion (A3) for the operator $n_{a}(t)$. By using the equations of motion for $a^{\dagger}$ and $a\left(a_{v}^{\dagger}\right.$ and $a_{v}$ ) without the terms proportional to $2 a^{\dagger} a\left(2 a_{v}^{\dagger} a_{v}\right)$, the same equation (A3) [(A4)] is obtained. Thus, to derive the rigorous $n_{a}(t)$, one can tweak the terms proportional to $2 a^{\dagger} a$ in Eqs. (6).

For bosonic systems, one can similarly derive the master equations for $n_{a}(t)$ and $n_{a_{v}}(t)$. They coincide with Eqs. (A3) and (A4), respectively, with only the replacement $1-n_{a}(s)-$ $n_{a_{v}}(s) \rightarrow 1+n_{a}(s)+n_{a_{v}}(s)$ because of different statistics. For the RWA coupling, there is a one-to-one correspondence between the master equations of the bosonic and fermionic systems.

\section{APPENDIX B}

The Heisenberg equations of motion for the creation and annihilation operators of intrinsic subsystems are obtained by commuting corresponding operator with $H$ :

$$
\begin{aligned}
& \frac{d}{d t} a_{\nu}^{\dagger}(t)=i \omega_{\nu} a_{\nu}^{\dagger}+\left(1-2 a_{v}^{\dagger} a_{v}\right) \frac{i}{\hbar} g_{\nu}\left(a^{\dagger}+a\right), \\
& \frac{d}{d t} a_{\nu}(t)=-i \omega_{\nu} a_{v}-\left(1-2 a_{\nu}^{\dagger} a_{\nu}\right) \frac{i}{\hbar} g_{\nu}\left(a^{\dagger}+a\right) .
\end{aligned}
$$

As in Eq. (6), we disregard here the terms proportional to $2 a_{v}^{\dagger} a_{v}$ (see Appendix A). The solution of Eq. (B1) is

$$
\begin{aligned}
a_{v}^{\dagger}(t)+a_{\nu}(t)= & {\left[e^{i \omega_{\nu} t} a_{\nu}^{\dagger}(0)+e^{-i \omega_{\nu} t} a_{\nu}(0)\right]+\frac{2 g_{v}}{\hbar \omega_{\nu}}\left\{-\left[a^{\dagger}(t)+a(t)\right]+\cos \left(\omega_{\nu} t\right)\left[a^{\dagger}(0)+a(0)\right]\right.} \\
& \left.+\int_{0}^{t} \cos \left[\omega_{\nu}(t-\tau)\right] \frac{d}{d \tau}\left[a^{\dagger}(\tau)+a(\tau)\right] d \tau\right\} .
\end{aligned}
$$

Substituting Eq. (B2) into Eq. (6) and eliminating the bath variables from the equations of motion for the collective subsystem, we obtain a set of Langevin-type integro-differential stochastic dissipative equations:

$$
\begin{aligned}
\frac{d}{d t}\left[a^{\dagger}(t)+a(t)\right] & =i \omega\left[a^{\dagger}(t)-a(t)\right]-i \frac{d}{d t}\left[a^{\dagger}(t)-a(t)\right] \\
& =\Omega\left[a^{\dagger}(t)+a(t)\right]+\int_{0}^{\infty} K(t-\tau) \frac{d}{d \tau}\left[a^{\dagger}(\tau)+a(\tau)\right] d \tau+F(t)+K(t)\left[a^{\dagger}(0)+a(0)\right],
\end{aligned}
$$

with the renormalized frequency

$$
\Omega=\omega-2 \sum_{\nu} \frac{2 g_{v}^{2}}{\hbar^{2} \omega_{v}}
$$

dissipative kernel

$$
K(t-\tau)=2 \sum_{\nu} \frac{2 g_{\nu}^{2}}{\hbar^{2} \omega_{\nu}} \cos \left[\omega_{\nu}(t-\tau)\right],
$$

and random force

$$
F(t)=\sum_{v} F_{v}(t)=\frac{2}{\hbar} \sum_{\nu} g_{v}\left[e^{i \omega_{v} t} a_{v}^{\dagger}(0)+e^{-i \omega_{v} t} a_{v}(0)\right] .
$$

In Eqs. (B3), the operator $F(t)$ plays a role of random force and depends on the initial conditions for the internal subsystem. The operators $F_{v}(t)$ are usually identified in statistical physics with fluctuations because of the uncertainty in the initial conditions for heat-bath operators $a_{\nu}^{\dagger}(0)$ and $a_{\nu}(0)$. Note that, in the case of bosonic systems, the combinations $a^{\dagger}(t)+a(t)$ and $i\left[a(t)-a^{\dagger}(t)\right]$ of creation and annihilation operators are the analogy of real coordinates and momentum.

To find a solution of Eqs. (B3), we apply the Laplace transform:

$$
G(s)=\frac{1}{s}[-\omega R(s)+G(0)], \quad R(s)=\frac{1}{s}[R(0)+\Omega G(s)+s K(s) G(s)+F(s)],
$$


where $G(s)=\mathcal{L}\left\{a^{\dagger}(t)+a(t)\right\}, R(s)=-i \mathcal{L}\left\{a^{\dagger}(t)-a(t)\right\}, K(s)=\mathcal{L}\{K(t)\}$, and

$$
F(s)=\mathcal{L}\{F(t)\}=\frac{2}{\hbar} \sum_{\nu} g_{v}\left[\frac{a_{\nu}^{\dagger}(0)}{s-i \omega_{\nu}}+\frac{a_{\nu}^{\dagger}(0)}{s-i \omega_{\nu}}\right]
$$

Solving the set of algebraic equations (B7), we find

$$
G(s)=\frac{s G(0)-\omega[R(0)+F(s)]}{s^{2}+\omega \Omega+s \omega K(s)}, \quad R(s)=\frac{\Omega G(0)+s[R(0)+G(0) K(s)+F(s)]}{s^{2}+\omega \Omega+s \omega K(s)} .
$$

The Laplace transforms of $a^{\dagger}(t)$ and $a(t)$ are

$$
\begin{aligned}
a^{\dagger}(s)= & a^{\dagger}(0) \frac{2 s+i(\omega+\Omega)+i s K(s)}{2\left[s^{2}+\omega \Omega+s \omega K(s)\right]}+a(0) \frac{i(\Omega-\omega)+i s K(s)}{2\left[s^{2}+\omega \Omega+s \omega K(s)\right]} \\
& +\sum_{\nu} \frac{g_{\nu}}{\hbar}\left[a_{\nu}^{\dagger}(0) \frac{i(s+i \omega)}{2\left(s+i \omega_{\nu}\right)\left[s^{2}+\omega \Omega+s \omega K(s)\right]}+a_{\nu}(0) \frac{i(s+i \omega)}{2\left(s-i \omega_{\nu}\right)\left[s^{2}+\omega \Omega+s \omega K(s)\right]}\right], \\
a(s)= & a^{\dagger}(0) \frac{-i(\Omega-\omega)-i s K(s)}{2\left[s^{2}+\omega \Omega+s \omega K(s)\right]}+a(0) \frac{2 s-i(\omega+\Omega)-i s K(s)}{2\left[s^{2}+\omega \Omega+s \omega K(s)\right]} \\
& +\sum_{\nu} \frac{g_{\nu}}{\hbar}\left[a_{\nu}^{\dagger}(0) \frac{-i(s-i \omega)}{2\left(s+i \omega_{\nu}\right)\left[s^{2}+\omega \Omega+s \omega K(s)\right]}+a_{\nu}(0) \frac{-i(s-i \omega)}{2\left(s-i \omega_{\nu}\right)\left[s^{2}+\omega \Omega+s \omega K(s)\right]}\right] .
\end{aligned}
$$

The explicit solutions for the originals are

$$
\begin{aligned}
& a^{\dagger}(t)=a^{\dagger}(0) A^{*}(t)+a(0) B(t)+\sum_{v} \frac{g_{v}}{\hbar} a_{v}^{\dagger}(0) M_{v}^{*}\left(t, w_{v}\right)+\sum_{v} a_{v}(0) \frac{g_{v}}{\hbar} N_{v}\left(t, w_{v}\right), \\
& a(t)=a(0) A(t)+a^{\dagger}(0) B^{*}(t)+\sum_{v} \frac{g_{v}}{\hbar} a_{v}^{\dagger}(0) N_{v}^{*}\left(t, w_{v}\right)+\sum_{v} a_{v}(0) \frac{g_{v}}{\hbar} M_{v}\left(t, w_{v}\right),
\end{aligned}
$$

where the time-dependent coefficients are denoted as follows:

$$
\begin{aligned}
A(t) & =\frac{1}{2} \mathcal{L}^{-1}\left\{\frac{2 s-i(\omega+\Omega)-i s K(s)}{2\left[s^{2}+\omega \Omega+s \omega K(s)\right]}\right\}, \\
B(t) & =\frac{1}{2} \mathcal{L}^{-1}\left\{\frac{i(\Omega-\omega)+i s K(s)}{2\left[s^{2}+\omega \Omega+s \omega K(s)\right]}\right\}, \\
M\left(t, w_{\nu}\right) & =\frac{1}{2} \mathcal{L}^{-1}\left\{\frac{-i(s-i \omega)}{2\left(s-i \omega_{\nu}\right)\left[s^{2}+\omega \Omega+s \omega K(s)\right]}\right\}, \\
N\left(t, w_{\nu}\right) & =\frac{1}{2} \mathcal{L}^{-1}\left\{\frac{i(s+i \omega)}{2\left(s-i \omega_{\nu}\right)\left[s^{2}+\omega \Omega+s \omega K(s)\right]}\right\} .
\end{aligned}
$$

So, one can write the time-dependent operator of the occupation number:

$$
\begin{aligned}
a^{\dagger}(t) a(t)= & a^{\dagger}(0) a(0) A^{*}(t) A(t)+a(0) a^{\dagger}(0) B^{*}(t) B(t) \\
& +\sum_{v} \frac{g_{v}^{2}}{\hbar^{2}} a_{v}^{\dagger}(0) a(0)_{v} M^{*}\left(t, w_{v}\right) M\left(t, w_{v}\right)+\sum_{v} \frac{g_{v}^{2}}{\hbar^{2}} a_{v}(0) a^{\dagger}(0)_{v} N^{*}\left(t, w_{v}\right) N\left(t, w_{v}\right) .
\end{aligned}
$$

To find the explicit expressions for the occupation number, it is convenient to introduce the spectral density $\rho(w)$ of the heat-bath excitations, which allows us to replace the sum over different two-level systems $v$ by the integral over the frequency: $\sum_{v} \cdots \rightarrow \int_{0}^{\infty} d w \rho(w) \cdots$. Let us consider the following spectral function [2]:

$$
\frac{g_{v}^{2}}{\hbar^{2} w_{v}} \rightarrow \frac{\rho(w) g^{2}(w)}{\hbar^{2} w}=\frac{1}{\pi} g_{0} \frac{\gamma^{2}}{\gamma^{2}+w^{2}},
$$

where the memory time $\gamma^{-1}$ of the dissipation is the inverse of the bandwidth of the heat-bath excitations which are coupled to the collective system. This is the Ohmic dissipation with the Lorentzian cutoff (Drude dissipation). The relaxation time of the heat bath should be much less than the characteristic collective time; i.e., $\gamma \gg \omega$. Employing Eq. (B11), we obtained the expressions for the dissipative kernel:

$$
K(t)=\frac{g_{0} \gamma^{2}}{\pi} \int_{0}^{\infty} d w \frac{\cos (w t)}{\gamma^{2}+w^{2}}=2 g_{0} \gamma e^{-\gamma t},
$$


and

$$
K(s)=\frac{2 g_{0} \gamma}{s+\gamma}
$$

This type of spectral function and dissipative kernel leads to expressions (7)-(9) for the occupation number.

The fluctuation-dissipation relations connect the dissipation of a collective subsystem and the fluctuations of random forces. These relations express the nonequilibrium behavior of the system in terms of equilibrium or quasi-equilibrium characteristics. They ensure that the system approaches the equilibrium state. We consider the initial distribution of bath fermionic operators $a_{\nu}^{\dagger}(0)$ and $a_{\nu}(0)$. For the correlation of the random force one can obtain

$$
\left\langle\left\langle F_{v}(t)\right\rangle\right\rangle=\left\langle\left\langle F_{\nu}(t) F_{\mu}(t)\right\rangle\right\rangle=0,
$$

and

$$
\left\langle\left\langle F_{\nu}(t) F_{v}(\tau)\right\rangle\right\rangle=\frac{4 g_{v}^{2}}{\hbar^{2}}\left[e^{i \omega_{v}(t-\tau)}\left\langle\left\langle a_{\nu}^{\dagger}(0) a_{v}(0)\right\rangle\right\rangle+e^{-i \omega_{v}(t-\tau)}\left\langle\left\langle a_{v}(0) a_{v}^{\dagger}(0)\right\rangle\right\rangle\right] .
$$

Using these expressions, one can get the fluctuation dissipation relations

$$
K(t-\tau)=\frac{1}{2} \sum_{\nu}\left\langle\left\langle F_{\nu}(t) F_{\nu}(\tau)+F_{v}(\tau) F_{v}(t)\right\rangle\right\rangle .
$$

Here, the symbol $\langle\langle\cdots\rangle\rangle$ denotes the average over the bath. Fulfillment of the fluctuation-dissipation relations means that we have correctly defined the dissipative kernels in the non-Markovian equations of motion.

[1] A. O. Caldeira and A. J. Leggett, Physica A 121, 587 (1983); Ann. Phys. (NY) 149, 374 (1983).

[2] K. Lindenberg and B. J. West, The Nonequilibrium Statistical Mechanics of Open and Closed Systems (VCH Publishers, Inc., New York, 1990); Phys. Rev. A 30, 568 (1984).

[3] F. Haake and R. Reibold, Phys. Rev. A 32, 2462 (1985).

[4] P. Schramm, R. Jung, and H. Grabert, Phys. Lett. A 107, 385 (1985).

[5] P. Talkner, Ann. Phys. (NY) 167, 390 (1986).

[6] V. V. Dodonov and V. I. Man'ko, Density Matrices and Wigner Functions of Quasiclassical Quantum Systems, Proc. Lebedev Phys. Inst. of Sciences, Vol. 167, edited by A. A. Komar (Nova Science, Commack, 1987).

[7] A. J. Leggett, S. Charkravarty, A. T. Dorsey, M. P. A. Fisher, A. Garg, and W. Zwerger, Rev. Mod. Phys. 59, 1 (1987).

[8] H. Grabert, P. Schramm, and G.-L. Ingold, Phys. Rep. 168, 115 (1988).

[9] G. W. Ford, J. T. Lewis, and R. F. O’Connell, Phys. Rev. A 37, 4419 (1988).

[10] A. V. Shelest, The Bogoliubov Method in Dynamic Theory of Kinetic Equations (Nauka, Moscow, 1990).

[11] C. W. Gardiner, Quantum Noise (Springer, Berlin, 1991).

[12] H. J. Carmichael, An Open System Approach to Quantum Optics (Springer, Berlin, 1993).

[13] B. L. Hu, J. P. Paz, and Y. Zhang, Phys. Rev. D 45, 2843 (1992); 47, 1576 (1993).

[14] A. Isar, A. Sandulescu, H. Scutaru, E. Stefanescu, and W. Scheid, Int. J. Mod. Phys. E 3, 635 (1994).

[15] V. V. Dodonov, O. V. Man'ko, and V. I. Man'ko, J. Russ. Laser Res. 16, 1 (1995).

[16] J. J. Halliwell and T. Yu, Phys. Rev. D 53, 2012 (1996).
[17] D. Zubarev, V. Morozov, and G. Röpke, Statistical Mechanics of Nonequilibrium Processes (Akademie Verlag, Berlin, 1997), Vol. II.

[18] U. Weiss, Quantum Dissipative Systems (Wold Scientific, Singapore, 1999).

[19] H. P. Breuer and F. Petruccione, Theory of Open Quantum Systems (Oxford University Press, 2002).

[20] Z. Kanokov, Yu. V. Palchikov, G. G. Adamian, N. V. Antonenko, and W. Scheid, Phys. Rev. E 71, 016121 (2005); Yu. V. Palchikov, Z. Kanokov, G. G. Adamian, N. V. Antonenko, and W. Scheid, ibid. 71, 016122 (2005).

[21] G. G. Adamian, N. V. Antonenko, Z. Kanokov, and V. V. Sargsyan, Theor. Math. Phys. 145, 1443 (2005).

[22] V. V. Sargsyan, Z. Kanokov, G. G. Adamian, and N. V. Antonenko, Phys. Rev. C 77, 024607 (2008).

[23] V. V. Sargsyan, Z. Kanokov, G. G. Adamian, and N. V. Antonenko, Phys. Part. Nucl. 41, 175 (2010).

[24] R. A. Kuzyakin, V. V. Sargsyan, G. G. Adamian, and N. V. Antonenko, Phys. Rev. A 83, 062117 (2011); 84, 032117 (2011).

[25] G. Ritschel and A. Eisfeld, J. Chem. Phys. 141, 094101 (2014)

[26] M. W. Y. Tu and W.-M. Zhang, Phys. Rev. B 78, 235311 (2008).

[27] M. Rigol, Phys. Rev. Lett. 112, 170601 (2014).

[28] D. Lacroix, V. V. Sargsyan, G. G. Adamian, and N. V. Antonenko, Eur. Phys. J. B 88, 89 (2015).

[29] H. Dekker, Physica A 144, 453 (1987); R. Egger, H. Grabert, and U. Weiss, Phys. Rev. E 55, R3809 (1997).

[30] W. T. Strunz, Phys. Lett. A 224, 25 (1996).

[31] L. Diosi, N. Gisin, and W. T. Strunz, Phys. Rev. A 58, 1699 (1998).

[32] W. T. Strunz, L. Diosi, and N. Gisin, Phys. Rev. Lett. 82, 1801 (1999).

[33] W. T. Strunz, New J. Phys. 7, 91 (2005). 
[34] J. Piilo, S. Maniscalco, K. Härkönen, and K.-A. Suominen, Phys. Rev. Lett. 100, 180402 (2008).

[35] K. Wen, F. Sakata, Z.-X. Li, X.-Z. Wu, Y.-X. Zhang, and S.-G. Zhou, Phys. Rev. Lett. 111, 012501 (2013).

[36] J. Shao, J. Chem. Phys. 120, 5053 (2004).

[37] D. Lacroix, Phys. Rev. A 72, 013805 (2005).

[38] D. Lacroix, Phys. Rev. E 77, 041126 (2008).
[39] G. Hupin and D. Lacroix, Phys. Rev. C 81, 014609 (2010).

[40] H.-P. Breuer, Eur. Phys. J. D 29, 105 (2004).

[41] V. V. Sargsyan, G. G. Adamian, N. V. Antonenko, and D. Lacroix, Phys. Rev. A 90, 022123 (2014).

[42] J. P. Blaizot and G. Ripka, Quantum Theory of Finite Systems (MIT Press, Cambridge, 1986). 